\title{
Bilateral thalamic infarction secondary to artery of Percheron occlusion: A rare cause of acute coma
}

\author{
James E. Brady ${ }^{* 1}$, Keith Brown ${ }^{1}$, Raqiya Shoaib ${ }^{1}$, David Roshal ${ }^{2}$ \\ ${ }^{1}$ Rowan School of Osteopathic Medicine, Jefferson Health System, Stratford, New Jersey, United States \\ 2 Jefferson Health New Jersey, Department of Neurology, Washington Township, New Jersey, United States
}

Received: December 19, 2020

Accepted: February 9, 2021

Online Published: February 26, 2021

DOI: $10.5430 /$ crim.v7n 4 p17

URL: https://doi.org/10.5430/crim.v7n4p17

\begin{abstract}
An occlusion of the artery of Percheron (AOP) is a rare clinical condition that remains a diagnostic challenge for physicians given its variable presentation. This infarction is a result of an anatomical variant of a single vessel arising from the posterior cerebral artery. Occlusion of the AOP results in variable levels of alteration of consciousness, vertical gaze palsy, and memory impairment. Unfortunately, due to this variable presentation these strokes are often missed. We report a case of a 71 year-old-female who presented with sudden onset of unresponsiveness who was subsequently diagnosed with bilateral thalamic infarction with midbrain involvement secondary to AOP occlusion. Delayed diagnosis can result in poor neurologic outcomes as demonstrated in this case making it imperative to bring further awareness to this clinical syndrome.
\end{abstract}

Key Words: Bilateral thalamic stroke, Cerebrovascular accident, Coma, Artery of Percheron, Stroke

\section{INTRODUCTION}

The artery of Percheron (AOP) is a rare anatomical variant in which a single branch from the posterior cerebral artery supplies the bilateral paramedian thalami and midbrain. Typically, the thalami and midbrain are supplied from the thalamoperforators from the posterior communicating arteries and the $\mathrm{P} 1$ and $\mathrm{P} 2$ segments of the posterior cerebral arteries. ${ }^{[1]}$ It is approximated that strokes involving the AOP account for only $0.1 \%-2 \%$ of all ischemic strokes, but the anatomic variant is estimated to be present in $4 \%-11 \%$ of the general population. ${ }^{[2,3]}$ Clinical features such as variable levels of consciousness, vertical gaze palsy, oculomotor disturbances, hemiplegia, cerebellar ataxia, and movement disorders have all been described. ${ }^{[2]}$ The clinical features of this stroke are very complex and the presentation variability often leads to a delay in diagnosis with a large differential diagnosis. Awareness of this syndrome may prompt more urgent diagnostic work-up leading to thrombolysis which could improve survival and functional recovery. ${ }^{[4]}$ We describe a case of a patient who presented with a witnessed sudden unresponsiveness due to occlusion of the artery of Percheron.

\section{Case presentation}

Our patient is a 71 year-old female who presented to the emergency department after she became completely unresponsive while eating dinner with her family. The family reported that she was in her usual state of health with no prodrome prior to this episode, which was described as a sudden comatose state. The patient has a past medical history of hypertension, asthma, arthritis, and a previous aortic valve replacement due to infective endocarditis complicated by a

\footnotetext{
* Correspondence: James E. Brady; Email: bradyj@ rowan.edu; Address: nternal Medicine, 42 E Laurel Road, Suite 3100. Stratford, New Jersey, 08084, United States.
} 
$1.2 \mathrm{~cm}$ vegetation requiring implantation of a 25 millimeter $(\mathrm{mm})$ bovine pericardium tissue valve several years prior.

On arrival to the emergency department she was intubated by emergency medicine physicians for protection of her airway with a Glasgow Coma Scale (GCS) of 3. Her vital signs were remarkable for an initial blood pressure of 239/185. Her physical examination revealed an intubated patient with $3 / 6$ systolic ejection murmur, $2+$ pitting edema in her lower extremities bilaterally. She remained stuporous off sedation, not following commands or opening eyes to pain or stimulation. Her pupils were asymmetric at 4 millimeter $(\mathrm{mm})$ in the right pupil and $2 \mathrm{~mm}$ in the left pupil. She did withdrawal to painful stimuli in her upper and lower extremities symmetrically bilaterally and demonstrated a positive cough and gag reflex.

The patients admission lab work which included a complete blood count (CBC), basic metabolic panel (BMP), prothrombin (PT), partial thromboplastin time (PTT), international normalized ratio (INR), and urine drug screen were all within normal limits. A computerized tomography (CT) of the brain as well as a CT angiogram of the head and neck were negative for acute intracranial abnormality or hemodynamically signif- icant stenosis or vascular occlusion. A magnetic resonance imaging (MRI) of the brain was performed the following day and demonstrated acute bilateral thalamic infarcts with midbrain extension suggestive of occlusion of the artery of Percheron (see Figure 1). An echocardiogram was done and negative for left ventricular thrombus with hyperdynamic left ventricular ejection fraction estimated to be 70\%-75\%. A video electroencephalogram (EEG) was done revealing diffuse voltage suppression and background slowing with no evidence of clinical seizures. There was no evidence of arrhythmia observed on her electrocardiogram or continuous cardiac monitoring.

Throughout the patient's hospital course she did not regain consciousness, however she did eventually move her extremities spontaneously. She failed her extubation trial from the ventilator and required tracheostomy and percutaneous endoscopic gastrostomy (PEG) tube. She was started on aspirin monotherapy and modafinil which she would continue as an outpatient. At the conclusion of her hospital stay, the patient was able to be discharged to a long term care facility for long term medical care at the decision of her immediate family members.

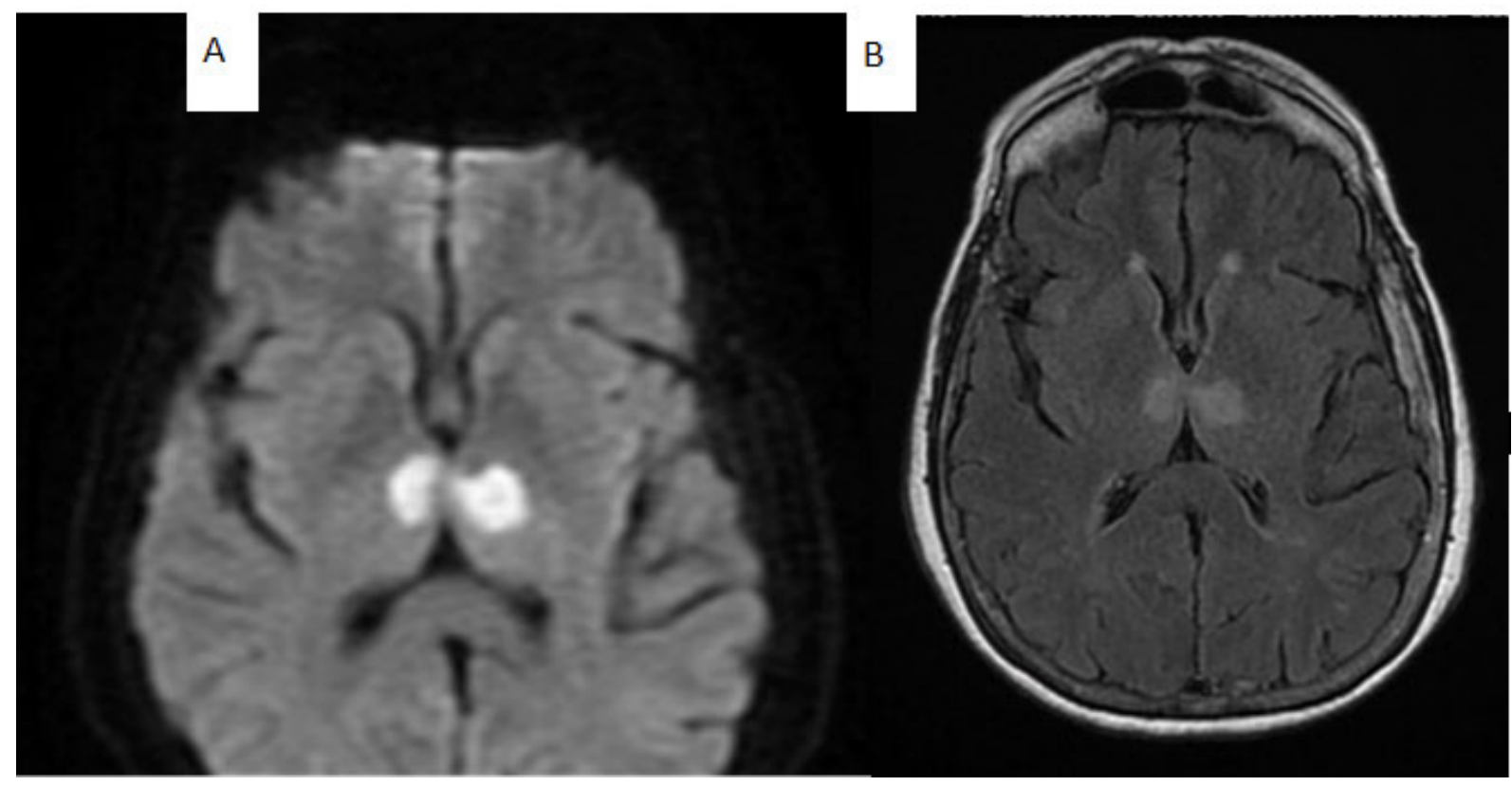

Figure 1. (A) Diffusion-weighted axial MRI brain showing diffusion restriction at the level of the bilateral paramedian thalami. (B) T2/FLAIR signal weighted axial MRI brain demonstrating hyperintensity at the level of the bilateral paramedian thalami

\section{DiscuSsion}

The thalamic blood supply and vascular syndromes are extremely complex as first studied in 1874 and further simplified throughout the literature over the course of a century. ${ }^{[5]}$
Percheron described possible variations of the paramedian thalamic-mesencephalic arterial supply, one being an uncommon variant of a single artery from the P1 segment. ${ }^{[6]}$ The artery of Percheron was definitively defined in 1973, and the 
rare occlusion of this posterior circulation artery results in bilateral paramedian thalamic infarction. The most common etiology of this particular stroke variant is thought to be cardioembolic in nature. ${ }^{[2,4]}$ Our patient did not demonstrate evidence of arrhythmias throughout her hospital stay, and echocardiogram findings were negative for septal defects, but a prehospital episode of paroxysmal arrhythmia cannot be excluded.

The thalamus plays an important role in regulating consciousness and bilateral thalamic injuries result in altered levels of consciousness that are variable in presentation. ${ }^{[1]}$ More commonly, diagnosis is made clinically and requires a high index of suspicion on part of the physician. ${ }^{[2]}$ In a study by Weidauer et al., sudden onset of somnolence was the most common neurological symptom seen in $87 \%$ of patients $(\mathrm{n}=17)$ upon presentation for medical care who subsequently had bilateral thalamic paramedian infarcts. This was followed by hemisyndromes, cognitive deficits, oculomotor nerve palsy, vertical gaze palsy, and aphasia. ${ }^{[7]}$ Due to this variability in presentation the differential diagnosis is broad with AOP occlusion, top of the basilar artery syndrome, and deep cerebral venous thrombosis as the leading differential diagnosis. ${ }^{[1]}$

Unfortunately, traditional vascular CT angiography does not routinely demonstrate the small perforating AOP vessel as evident in this patient in which a head CT and CT angiography revealed no evidence of infarction or occlusion. ${ }^{[2]}$ This neuroradiographic challenge exists due to the small vessel size of the infrequently encountered artery. ${ }^{[8]}$ Brain MRI is the test of choice for identification of AOP occlusion and is the most specific imaging modality available. ${ }^{[3]}$ Lazarro et al reported a distinct pattern of V-shaped hyperintensity on axial fluid-attenuated inversion recovery (FLAIR) and/or diffusion weighted imaging (DWI) on MRI that is also present in the midbrain of patients with occlusion of the AOP and has a sensitivity of $67 \% .{ }^{[3,9]}$ However, given the absence of findings on initial CT, MRI is delayed and many patients remain undiagnosed for a significant amount of time. ${ }^{[10]}$ In a study by Staum et al, an average time delay of 1.9 days between presentation and diagnostic imaging was observed. ${ }^{[7]}$

The variable nature of presentation of an AOP infarct makes diagnosis difficult. However, when the presentation of AOP infarct is compared to the presentation of infarct in other more common vessels there are some unique features that can help guide the clinician in at least placing AOP infarct on their differential. This differentiation would allow for more expedient diagnosis and evaluation for intervention. The most commonly occluded vessel in acute ischemic stroke is the middle cerebral artery (MCA), which classically presents

Published by Sciedu Press as contralateral hemiparesis, ipsilateral facial paralysis, and sensory loss in the face and upper extremity and a horizontal gaze palsy. ${ }^{[11]}$ When compared with patients in a study by Lazzaro et al who had confirmed AOP infarcts revealing lesions in the bilateral paramedian thalamus and $43 \%$ had rostral midbrain involvement. Involvement of bilateral paramedian thalamus leads to the recognizable triad of altered mental status, vertical gaze palsy, and amnesia. Rostral midbrain involvement may lead to other oculomotor disturbances, hemiplegia, cerebellar ataxia, and movement disorders. ${ }^{[9]}$ If physical exam findings are suggestive of paramedian thalamic or rostral midbrain involvement then a CT angiogram may be diagnostic. However, if the CT angiogram is negative, this may suggest embolic occlusion of the distal basilar artery which is more difficult to detect on routine imaging. At this juncture, an MRI should be obtained urgently as it is capable of providing crucial diagnostic information and lead to more rapid neurointervention. ${ }^{[9]}$

A case report published by Kostanian et al is one of few, if not the only, cases of angiographically proven and treated AOP occlusion. They were able to use clinical exam findings as described previously to narrow their differential to include rostral brainstem involvement. The facility in the study had neurointerventional capabilities and cerebral angiogram was performed revealing a small filling defect was seen in the area of the basilar artery tip/proximal P1 segment of the right posterior cerebral artery with an aplastic right P1 segment. Intravascular tissue plasminogen activator (tPA) and periprocedural anticoagulation with intravenous heparin was used to achieve recanalization of the AOP. The patient's neurologic deficits of left sided paresis, stupor, ptosis, internuclear ophthalmoplegia, and gaze preference did significantly improve with this intervention. ${ }^{[6]}$ With this evidence, facilities capable of neurointerventional procedures should expediently evaluate these patients for neuroendovascular intervention to provide these patients with the most favorable opportunity for neurologic recovery.

Due to the rare anatomic variant and variability of presenting features, physicians are often unaware of this type of stroke and its management. ${ }^{[8]}$ Standard of care for the treatment exists for management of ischemic stroke and is contingent on timing of presentation, location of the lesion, and evaluation for tPA. ${ }^{[8]}$ As demonstrated in a previous case report, guideline directed management with tPA in a patient with AOP occlusion resulted in a relatively favorable neurological outcome. ${ }^{[6]}$ Long term outcomes for patients with bilateral paramedian thalamic infarction remain variable, but persistent memory dysfunction and decreased level of consciousness seem to be common outcomes in follow up in those who do not receive intervention due to the time delay to diagnosis. 
In a previous study, only one third of patients with AOP were discharged directly to home, and most have significant persistent neurological deficits as did our patient. ${ }^{[7]}$ It is our hope that this case report will continue to bring awareness to this condition and improve patient neurologic outcomes.

\section{Conclusion}

Clinical variability seen in patients as a result of AOP occlusion continues to present a diagnostic challenge for physicians. Lack of diagnostic symptoms and lack of findings on initial CT often delay prompt administration of time sensi- tive tPA. This delay most certainly leads to negative long term outcomes as we have demonstrated in this patient. It is our hope that this case will allow clinicians to continue to maintain a large differential diagnosis, but consider AOP occlusion in patients who present with nonfocal and variable neurological symptoms which will allow a low threshold for a timely and diagnostic MRI study. This clinical acumen will likely lead to improved outcomes in these patients diagnosed with AOP infarctions.

\section{CONFlicts OF INTEREST Disclosure}

The authors have declared no conflicts of interest.

\section{REFERENCES}

[1] Agarwal S, Chancellor B, Howard J. Clinical-radiographic correlates of artery of percheron infarcts in a case series of 6 patients. Journal of Clinical Neuroscience. 2019; 266-268. PMid:30472345. https://doi.org/10.1016/j.jocn.2018.11.030

[2] Caruso P, Manganotti P, Moretti R. Complex neurological symptoms in bilateral thalamic stroke due to percheron artery occlusion. Vascular Health and Risk Management. 2017; 13: 11-14. PMid:28053539. https://doi.org/10.2147/VHRM.S119395

[3] Garcia-graimshaw M, Peschard-franco M, Gutierrez-manjarrez F. Bilateral thalamic ischemic stroke secondary to occlusion of the artery of percheron. Cureus. 2018: 10(5): e2676. https://doi .org/10 .7759 /cureus 2676

[4] Hegde AN, Mohan S, Lath N, et al. Differential diagnosis for bilateral abnormalities of the basal ganglia and thalamus. Radiographics 2011; 31: 5-30. PMid:21257930. https://doi .org/10.1148/rg .311105041

[5] Schmahmann J. Vascular syndromes of the thalamus. Stroke. 2003; 34: 2264-2278. PMid:12933968. https://doi.org/10.1161/01
.STR.0000087786.38997.9E

[6] Konstanian V, Cramer SC. Artery of percheron thrombolysis. AJNR Am J Neuroradiol. 2007; 28: 870-871.

[7] Stamm B, Lineback C, Skolarus L, et al. Artery of percheron infarct: 12 cases and their complex clinical courses. The Neurohospitalist. 2018; 8(3): 141-145. PMid:29977445. https://doi.org/10.117 $7 / 1941874417748543$

[8] Kichloo A, Jamal S, Zain El-Amir, et al. Artery of percheron infarction: a short review. Journal of Investigative Medicine High Impact Case Reports. 2019; 7: 1-6. PMid:31394937. https://doi.org/ $10.1177 / 2324709619867355$

[9] Lazzaro N, Wright B, Castillo M, et al. Artery of percheron infarction: imaging patterns and clinical spectrum. AJNR Am J Neuroradiol. 2010; 31: 1283-1289. PMid:20299438. https://doi.org/10.3 174/ajnr.A2044

[10] Khanni J, Casale J, Koek A, et al. Artery of Percheron infarct: An acute diagnostic challenge with a spectrum of clinical presentations. 2018; 10(9): e3276. https://doi.org/10.7759/cureus . 3276

[11] Hui C, Tadi P, Patti L. (2020). Ischemic Stroke. StatPearls Publishing. 